\title{
Modified Nonparametric Weighted Feature Extraction Algorithm
}

\author{
Linlin Cui • Guosheng Li • Huiru Ren • Lei He • \\ Huajun Liao
}

Received: 27 December 2013 / Accepted: 26 May 2014 / Published online: 29 July 2014

(C) Indian Society of Remote Sensing 2014

\begin{abstract}
Nonparametric weighted feature extraction (NWFE) has been proven to be a powerful feature extraction tool for hyperspectral data classification with a weight function based on Euclidean distance (ED). In this paper, we propose a modified algorithm referred to as nonparametric weighted spectral pan-similarity measure feature extraction (NWSPMFE). In NWSPMFE, ED is replaced by the spectral pan-similarity measure, and the weight function is redefined in scatter matrices for NWFE. The performance of NWSPMFE is evaluated by comparing it with principal component analysis (PCA) and NWFE in terms of overall accuracy and Kappa analysis based on two experiment datasets. The overall classification accuracies of PCA, NWFE, and NWSPMFE for D.C. Mall and Indian Pine datasets are $0.942,0.949,0.961$ and $0.496,0.665,0.697$, respectively. However, NWSPMFE's runtime is slightly longer than that of NWFE.
\end{abstract}

Keywords Spectral pan-similarity measure (SPM) . Euclidean distance (ED) · Nonparametric weighted spectral pan-similarity measure feature extraction (NWSPMFE) . Nonparametric weighted feature extraction (NWFE)

L. Cui $\cdot$ G. Li $(\bowtie) \cdot$ H. Ren $\cdot$ L. He $\cdot$ H. Liao

Institute of Geographic Sciences and Natural Resources Research, Chinese Academy of Sciences (CAS), Beijing, China

e-mail: ligscas@gmail.com

L. Cui $\cdot \mathrm{G} . \mathrm{Li} \cdot \mathrm{H}$. Ren $\cdot$ L. He $\cdot$ H. Liao

Key Laboratory of Coastal Wetland Biogeosciences, China Geologic

Survey, Qingdao 266071, China

L. Cui $\cdot$ H. Ren $\cdot$ L. He $\cdot$ H. Liao

University of Chinese Academy of Sciences, Beijing, China

\section{Introduction}

Classification is an important aspect of remote sensing data processing for many ecological and environmental studies. However, the Hughes phenomenon (Hughes, 1968) and singularity problems (Landgrebe, 2003) are usually found in hyperspectral data classification due to small training samples. Many classification algorithms and techniques have been developed to solve these problems and improve classification accuracy. Based on their specificity, these techniques can be divided into the following three categories (Landgrebe and Kuo 2002): (1) dimensionality reduction by feature extraction or feature selection (Landgrebe and Kuo 2002; Kuo, B.-C.and C.-H. Li 2005); (2) regularization of sample covariance matrix (Friedman, 1989; Rayens and Greene 1991); and (3) structurization of a true covariance matrix described by a small number of parameters (Raudys and Saudargiene, 1998).

Classification accuracy is determined based on many factors such as class separability, training sample size, dimensionality, and classifier type (Hsieh and David Landgrebe 1998). Many researchers indicated that dimensionality reduction is a key step of digital image classification (Tang et al., 2002; Sun et al., 2005;Tian et al., 2005;Das et al., 2006). Approaches to dimensionality reduction include feature selection and feature extraction (Young et al. 1986); we focus on the latter in this paper. Many feature extraction methods have been proposed in the past decades; such methods include principal component analysis (PCA), Fisher discriminant analysis (FDA), and independent component analysis, which are widely used. A new linear feature extraction technique called nonparametric weighted feature extraction (NWFE) (Kuo and David A Landgrebe 2002a;Kuo and Landgrebe 2004) was proposed recently. The image classification accuracy of NWFE is superior to that of FDA and nonparametric analysis (Kuo and David A Landgrebe 2002a;Kuo and Landgrebe 2004). 
The spectral similarity measure based on spectral feature is a key technique of many feature extraction methods. A hyperspectral image pixel is a column vector with a dimension that is equal to the number of bands. Therefore, spectral features mainly include spectral vector magnitude, direction, and information content, among others. The magnitude of a vector is the length of the vector, the direction of a vector is the measure of the angle it makes with a horizontal line, and the spectral information content is the measure of spectral variability, similarity, and discrimination (Chang, Chein-I 2000). Traditional spectral similarity metrics quantify only one of them, such as Euclidean distance (ED) (Chen et al., 2012), Mahalanobis distance (MD) (De Maesschalck et al., 2000), anisotropy-tunable distance (ATD) (Chen et al. 2012), Lance and Williams distance (LWD), Spectral Correlation Mapper (SCM) (van der Meer and Bakker 1997), Spectral Angle Mapper (SAM) (Chang, Chein-I 2000; Du et al., 2004;Chen et al., 2012), and spectral information divergence (SID) (Chang, Chein-I 2000;Du et al., 2004). ED, MD, ATD, and LWD primarily quantify magnitude differences, but they do not respond to direction and information content differences between two spectra. SCM and SAM are very responsive to differences in spectral shape (i.e., direction), but they do not respond to magnitude and information content differences. SID measures only the spectral information content differences between two spectra. Carvalho Júnior et al. (2011) indicated that the distance and direction (or shape) measures are complementary and need to be applied together. Sweet and James Norman (2003) pointed out that this single feature is not enough to fully capture spectral feature differences between two spectra. He also recommended the use of spectral similarity scale (SSS) to quantify magnitude and direction differences. Subsequently, other algorithms that mix two spectral features were also developed. Du et al. (2004) proposed a mixed measure, SID (TAN), by combining the SID and SAM. Naresh Kumar et al. (2011) proposed SIDSCA, by combining the spectral correlation angle (i.e., SCM) and SID. Robust spectral similarity metrics must capture more spectral differences. Kong et al. (2012) developed the spectral pan-similarity measure (SPM) to quantify magnitude, direction, and information content differences between two spectra. The results from SPM showed that it is more effective than the spectral similarity measure, taking into account only one (ED, SCM, SID) or two spectral features (SSS and SID (TAN)) in spectral discriminatory power and spectral identification uncertainty.

NWFE is used for dimensionality reduction in image classification. The most important parts of NWFE are weighted between-class and within-class scatter matrices and regularization (Kuo and David A Landgrebe 2002a). Determining or defining weight function is the critical step. The weight function based on the ED in NWFE is used to assign different weights to every sample to compute the weighted mean. However, ED measures only the magnitude differences between two spectra. As mentioned in the previous section, the spectral similarity metrics that consider more features can capture more differences. To fully capture the spectral differences, the modified algorithm, which is called the nonparametric weighted spectral pan-similarity measure feature extraction (NWSPMFE), replaces ED with SPM and redefines the weight function of the scatter matrices in NWFE. The performance of NWSPMFE in image classification is evaluated by comparing it with that of PCA and NWFE.

\section{Feature Extraction Methods}

NWFE was developed by Kuo and Landgrebe (Kuo and David A Landgrebe 2002a). In NWFE, the ED takes the following form (Kong et al. 2012):

$\mathrm{ED}(\mathbf{a}, \mathbf{b})=\sqrt{\frac{1}{N} \sum_{k=1}^{N}\left(\mathbf{a}_{k}-\mathbf{b}_{k}\right)^{2}}$

where $\mathrm{N}$ is the number of element in vectors a and $b$.

The core ideals of NWFE are to assign a different weight to every sample to compute the weighted means and to define nonparametric between-class and within-class scatter matrices. The between-class scatter matrix $\mathrm{S}_{\mathrm{b}}^{\mathrm{ED}}$ and the within-class scatter matrix $S_{\mathrm{w}}^{\mathrm{ED}}$ are given by

$\mathrm{S}_{\mathrm{b}}^{\mathrm{ED}}=\sum_{i=1}^{L} H_{i} \sum_{\substack{j \neq i \\ j \neq i}}^{L} \sum_{l=1}^{N_{i}} \frac{\lambda_{l}^{(i, j)}}{N_{i}}\left(\mathbf{x}_{l}^{(i)}-\mathbf{M}_{j}\left(\mathbf{x}_{l}^{(i)}\right)\right)\left(\mathbf{x}_{l}^{(i)}-\mathbf{M}_{j}\left(\mathbf{x}_{l}^{(i)}\right)\right)^{\mathrm{T}}$

And

$\mathrm{S}_{\mathrm{w}}^{\mathrm{ED}}=\sum_{i=1}^{L} H_{i} \sum_{l=1}^{N_{i}}\left(\mathbf{x}_{l}^{(i)}-\mathbf{M}_{i}\left(\mathbf{x}_{l}^{(i)}\right)\right)\left(\mathbf{x}_{l}^{(i)}-\mathbf{M}_{i}\left(\mathbf{x}_{l}^{(i)}\right)\right)^{\mathrm{T}}$

where $\mathbf{H}_{i}$ denotes the prior probability of class $i, \mathbf{N}_{i}$ is the number of training samples of the $i$ th class, $\boldsymbol{x}_{l}^{(i)}$ is the training sample in class $i$, and $\mathrm{T}$ is the transposition operator. The scatter matrix weight function $\lambda_{l}^{(i, j)}$ is defined by

$\lambda_{l}^{(i, j)}=\mathrm{ED}\left(\mathbf{x}_{l}^{(i)}, \mathbf{M}_{j}\left(\mathbf{x}_{l}^{(i)}\right)\right)^{-1} / \sum_{t=1}^{N_{i}} \operatorname{ED}\left(\mathbf{x}_{t}^{(i)}, \mathbf{M}_{j}\left(\mathbf{x}_{t}^{(i)}\right)\right)^{-1}$

Where

$\boldsymbol{M}_{j}\left(\boldsymbol{x}_{l}^{(i)}\right)=\sum_{k=1}^{\mathbf{N}_{j}} \boldsymbol{w}_{l k}^{(i, j)} \boldsymbol{x}_{k}^{(j)}$

$\boldsymbol{w}_{l k}^{(i, j)}=\mathrm{ED}\left(\boldsymbol{x}_{l}^{(i)}, \boldsymbol{x}_{k}^{(j)}\right)^{-1} / \sum_{t=1}^{\mathbf{N}_{j}} \operatorname{ED}\left(\boldsymbol{x}_{l}^{(i)}, \boldsymbol{x}_{k}^{(j)}\right)^{-1}$ 
$\boldsymbol{M}_{j}\left(\boldsymbol{x}_{l}^{(i)}\right)$ is the weighted mean of $\boldsymbol{x}_{l}^{(i)}$ in the $j$ th class. If the ED value between $\boldsymbol{x}_{l}^{(i)}$ and $\boldsymbol{M}_{j}\left(\boldsymbol{x}_{l}^{(i)}\right)$ is small, the scatter matrix weight $\boldsymbol{\lambda}_{l}^{(i, j)}$ will be close to 1 ; otherwise, $\boldsymbol{\lambda}_{l}^{(i, j)}$ will be close to 0 . Likewise, if the ED value between $\boldsymbol{x}_{l}^{(i)}$ and $\boldsymbol{x}_{k}^{(j)}$ is small, the weight $\boldsymbol{w}_{l k}^{(i, j)}$ will be close to 1 ; otherwise, $\boldsymbol{w}_{l k}^{(i, j)}$ will be close to 0 .

The purpose of NWFE is to find a linear mapping that can maximize the between-class scatter and minimize the within-class scatter. Therefore, the problem can be solved by finding eigenvalues $\lambda$ and eigenvectors $\mathbf{v}$ of the equation as follows:

$\boldsymbol{S}_{\mathrm{b}}^{\mathrm{ED}} \boldsymbol{v}=\lambda \boldsymbol{S}_{\mathrm{w}}^{\mathrm{ED}} \boldsymbol{v}$

To prevent the singularity of a matrix, many approaches have been developed (Friedman, 1989;Kuo et al., 2003;Kuo and Landgrebe 2004). In the current paper, a simple technique is used to regularize the within-class scatter matrix. Then, the within-class scatter matrix is written as

$\boldsymbol{S}_{\mathrm{w}}^{\mathrm{ED}}=0.5 \times \boldsymbol{S}_{\mathrm{w}}^{\mathrm{ED}}+0.5 \times \operatorname{diag}\left(\boldsymbol{S}_{\mathrm{w}}^{\mathrm{ED}}\right)$

where diag $\left(\boldsymbol{S}_{\mathrm{w}}^{\mathrm{ED}}\right)$ denotes the diagonal elements of matrix $\boldsymbol{S}_{\mathrm{w}}^{\mathrm{ED}}$.

As mentioned before, ED quantifies only magnitude differences between two spectra. To capture more accurate spectral differences, the modified algorithm, which is called NWSPMFE, replaced ED with SPM and redefined the weight function. Unlike with NWFE, only the scatter matrix weight function $\lambda_{l}^{(i, j)}$ needed to be redefined in NWSPMFE. The scatter matrix weight function $\boldsymbol{\lambda}_{l}^{(i, j)}$ in NWSPMFE is now defined by

$\boldsymbol{\lambda}_{l}^{(i, j)}=\operatorname{SPM}\left(\boldsymbol{x}_{l}^{(i)}, \boldsymbol{M}_{j}\left(\boldsymbol{x}_{l}^{(i)}\right)\right)^{-1} / \sum_{t=1}^{\mathbf{N}_{i}} \operatorname{SPM}\left(\boldsymbol{x}_{t}^{(i)}, \boldsymbol{M}_{j}\left(\boldsymbol{x}_{t}^{(i)}\right)\right)^{-1}$

Where

$\boldsymbol{M}_{j}\left(\boldsymbol{x}_{l}^{(i)}\right)=\sum_{k=1}^{\mathbf{N}_{j}} \boldsymbol{w}_{l k}^{(i, j)} \boldsymbol{x}_{k}^{(j)}$

$\boldsymbol{w}_{l k}^{(i, j)}=\operatorname{SPM}\left(\boldsymbol{x}_{l}^{(i)}, \boldsymbol{x}_{k}^{(j)}\right)^{-1} / \sum_{t=1}^{\mathbf{N}_{j}} \operatorname{SPM}\left(\boldsymbol{x}_{l}^{(i)}, \boldsymbol{x}_{k}^{(j)}\right)^{-1}$

Other equations of NWSPMFE are the same as NWFE.

The SPM is defined as (Kong et al. 2012)

$\operatorname{SPM}(\mathbf{a}, \mathbf{b})=\operatorname{SID}(\mathbf{a}, \mathbf{b}) \times \tan \left(\sqrt{\operatorname{ED}(\mathbf{a}, \mathbf{b})^{2}+\operatorname{SSD}(\mathbf{a}, \mathbf{b})^{2}}\right)$ where the spectral shape difference is given by

$\operatorname{SSD}(a, b)=((1-\operatorname{SCM}(a, b)) / 2)^{2}$

$\operatorname{SCM}(\mathbf{a}, \mathbf{b})=\left(\sum_{k=1}^{N}\left(\mathbf{a}_{k}-\overline{\mathbf{a}}\right)\left(\mathbf{b}_{k}-\overline{\mathbf{b}}\right)\right) /\left(\sqrt{\sum_{k=1}^{N}\left(\mathbf{a}_{k}-\overline{\mathbf{a}}\right)^{2}} \times \sqrt{\sum_{k=1}^{N}\left(\mathbf{b}_{k}-\overline{\mathbf{b}}\right)^{2}}\right)$

The SID is written as

$\operatorname{SID}(\mathbf{a}, \mathbf{b})=\sum_{k=1}^{N} \mathbf{p}_{k} \log \left(\frac{\mathbf{p}_{k}}{\mathbf{q}_{k}}\right)+\sum_{k=1}^{N} \mathbf{q}_{k} \log \left(\frac{\mathbf{q}_{k}}{\mathbf{p}_{k}}\right)$

Where $\mathbf{p}$ and $\mathbf{q}$ are probability measures of vectors a and $\mathbf{b}$, respectively. The probability measure $\mathbf{p}$ is defined as

$\mathbf{p}_{k}=\mathbf{a}_{k} / \sum_{i=1}^{N} \mathbf{a}_{i}$

\section{Materials and Experiment}

To evaluate the performance of NWSPMFE, two hyperspectral datasets were selected (https://engineering. purdue.edu/ biehl/multiSpec/). The first dataset is the Washington, D.C. Mall data, which was selected to represent an urban site. Hyperspectral Digital Imagery Collection Experiment data were acquired on August 23, 1995. The data set contains 210 bands in the $0.4 \mu \mathrm{m}$ to $2.4 \mu \mathrm{m}$ region of the visible and infrared spectrum. Some water absorption bands were discarded, leaving only 191 bands. Seven land use/land cover types were recorded in the Washington, D.C. Mall data; these include roofs, street, patch, grass, trees, water, and shadows. A $206 \times 307$ pixel subset of the Washington, D. C. Mall data and all 7 land use/land cover types were used in this paper. The second dataset is the Indian Pine AVIRIS (Airborne Visible/Infrared Imaging Spectrometer) hyperspectral data set. It consists of $145 \times 145$ pixels and 220 bands in the 0.4 to $2.5 \mu \mathrm{m}$ region of the visible and infrared spectrum with about two-thirds agriculture and one-third forest and other natural vegetation. The dataset was gathered on June 12, 1992 with a ground pixel size of $17 \mathrm{~m}$. For Indian Pine data, 16 land use/land cover types were available: Alfalfa, Bldg-Grass-Tree-Drives, Corn-notill, Corn-min, Corn, Grass/Pasture, Grass/Trees, Grass/Pasture-mowed, Haywindowed, Oats, Soybeans-notill, Soybeans-min, Soybeanclean, Stone-steel towers, Wheat, and Woods. All 16 classes were used in this paper.

To compare the performances of PCA, NWFE, and NWSPMFE, normal densities-based quadratic (QDC) (Webb, 2002; Duda et al., 2012), 2-nearest neighbor (2NN) (Hastie et al., 2009), and Parzen classifiers (Parzen, 1962) were used. The three classifiers were chosen for the following reasons: (1) These classifiers are based on perfect theories, widely used in satellite remote sensing data classification, and 
are classical classification methods. (2) These classifiers can be divided into two types: parametric classifiers and nonparametric classifiers. 2NN and Parzen classifiers are nonparametric classifiers, and QDC is the parametric classifier. One reason for choosing the three classifiers is that the probability density function is unknown. (3) Previous studies have shown that 2NN, Parzen, and QDC perform well (Pekalska et al., 2001; Pękalska and Duin, 2002; Paclık and Duin, 2003; Kuo and Landgrebe 2004 Kuo, B.-C.and C.-H. Li 2005; (Duin and Pekalska, 2007). (4) Kuo and David A Landgrebe (2002a)), Landgrebe and Kuo 2002 (Kuo and Landgrebe 2004) and (Kuo, B.-C.and C.-H. Li (2005)) have also successfully used the three classifiers to classify NWFE or Kernel-NWFE features. The three classifiers are described in PRTools (pattern recognition toolbox) (Duin, 2003). For each class in the two datasets, the training sample numbers of only Alfalfa, Grass/ Pasture-mowed, and Oats classes are 40, 25, and 15, respectively. Their testing sample numbers are the same as the training sample numbers. The training sample number of other classes for the two datasets is 40 , whereas the testing sample number is 60. According to previous research (Congalton, 1991; Foody, 1992, 2002; Liu et al., 2007; Ma and Redmond 1995; Smits et al., 1999; Stehman, 1997; Stehman and Czaplewski, 1998; Story and Congalton 1986), overall accuracy and Kappa analysis are used as the criteria to assess the accuracy of classifications of remotely sensed data.

Feature selection is also a key step in image classification; in other words, the number of extracted features is an important parameter. Mahalanobis, divergence, transformed divergence, Bhattacharya, and Jeffries-Matusita are widely used to obtain this parameter (Schowengerdt, 2006). Gong et al.(1992) indicated that Transformed Divergence and Jeffries-Matusita have similar performances. In the current paper, the mean and variance of the minimum value between transformed divergence and Jeffries-Matusita distances are used to determine the number of extracted features.

PCA was performed by using the ENVI Version 5.0 software. Programs of NWFE and NWSPMFE based on the ENVI/IDL software were developed for use in this paper. The classification was performed by using the pattern recognition toolbox, PRTools Version 3.2 (Duin, 2003), developed for use within Matlab.

\section{Experimental Results}

For a given hyperspectral dataset, the number of features is equal to $\mathrm{Y}(\mathrm{Y}=1,2,3, \ldots, \mathrm{m})$, where $\mathrm{m}$ equals the band number of the given hyperspectral dataset. If $Y=1$, then 1 represents the first feature band; if $Y=2$, then 2 is the first two feature bands, etc. Given the computational complexity of NWSPMFE, we computed the total runtime of NWSPMFE with that of NWFE on the same platform. For the D.C. Mall (a)

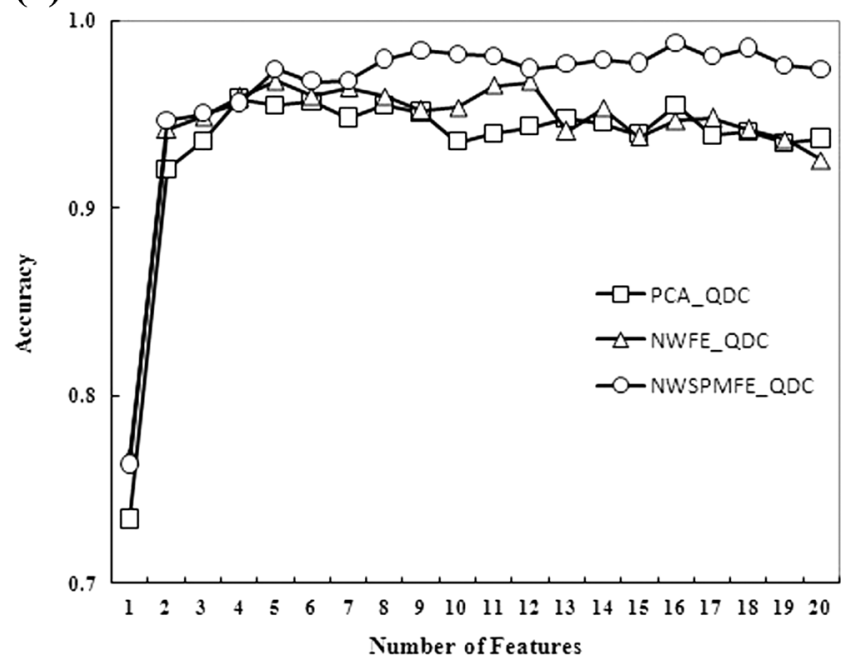

(b)

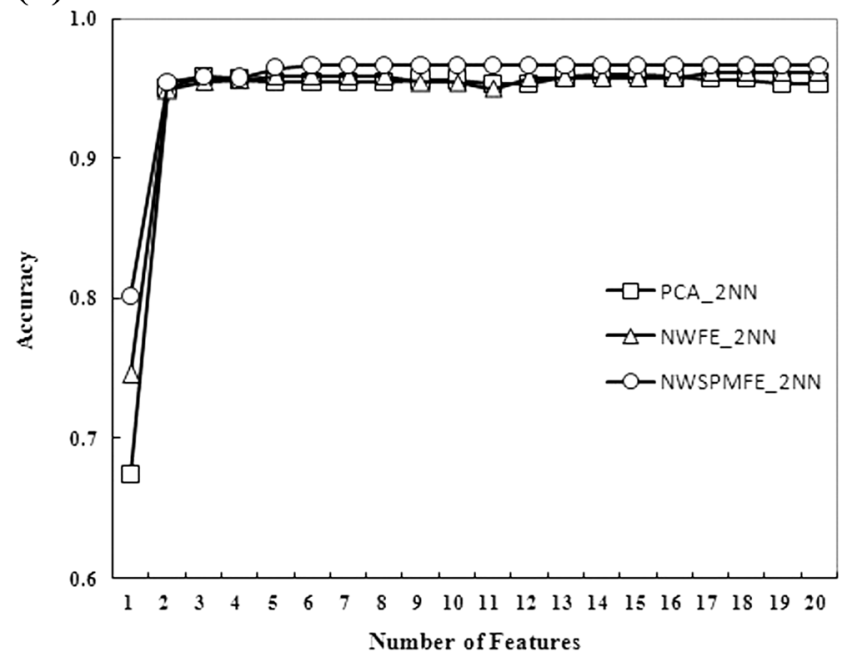

(c)

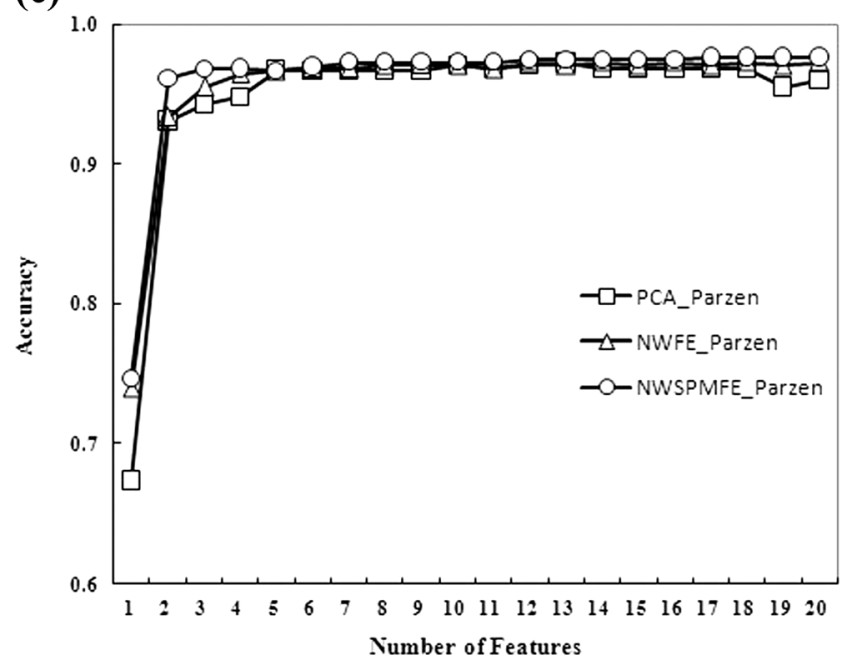

Fig. 1 Overall accuracy versus number of selected features for the three classifiers (D.C. Mall): (a) $Q D C$, (b) $2 \mathrm{NN}$, (c) Parzen 
Fig. 2 Mean and variance of the minimum value between transformed divergence and Jeffries-Matusita distances using 2-20 features (D.C. Mall)

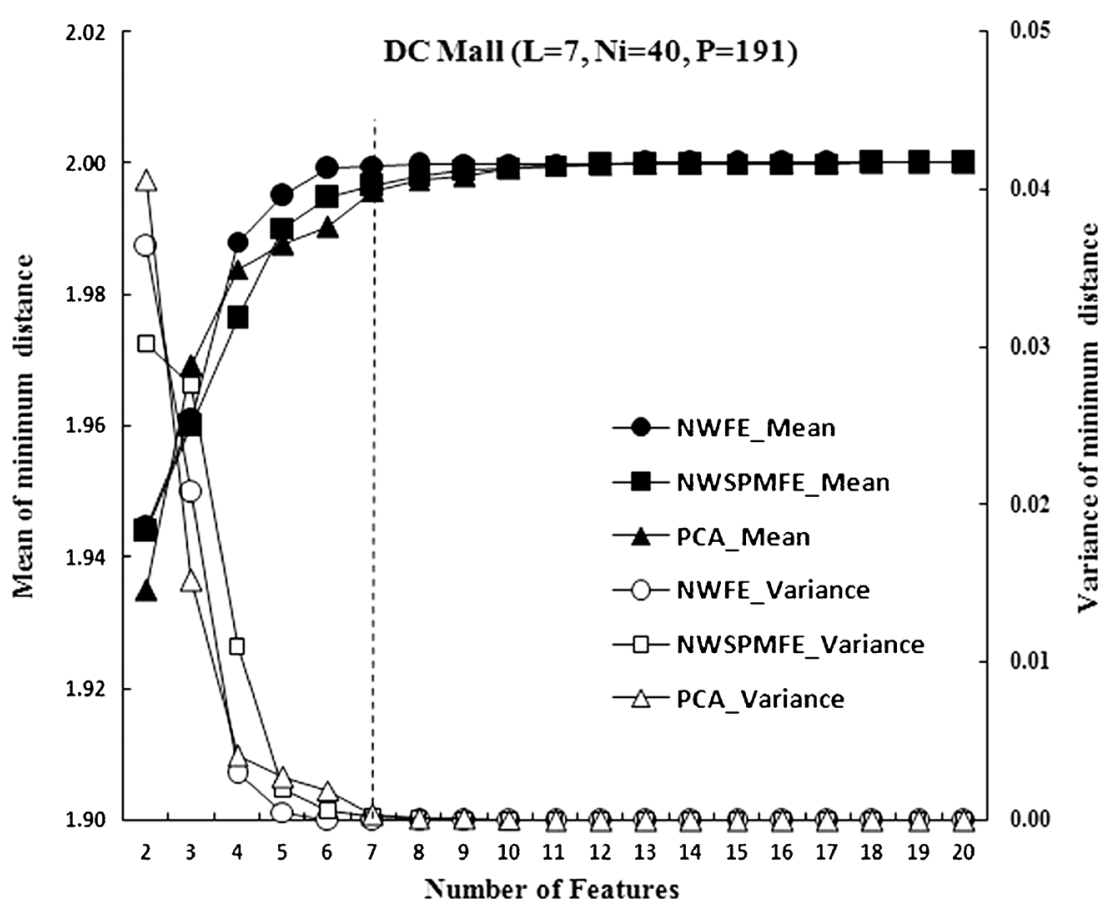

dataset, the extraction runtimes for 20 NWSPMFE and NWFE features are 110.56 and $102.43 \mathrm{~s}$, respectively. Similarly, for the Indian Pine dataset, the corresponding runtimes are 60.16 and $55.60 \mathrm{~s}$, respectively. Here, $\mathrm{L}$ is the number of classes, $\mathrm{Ni}$ indicates the number of training samples in each class, and $\mathrm{p}$ indicates the number of features extracted by PCA, NWFE or NWSPMFE.

\section{Results of D.C. Mall Dataset}

The normal densities-based QDC, 2NN, and Parzen classifiers are used to evaluate the performances of PCA, NWFE, and NWSPMFE. The overall accuracies using 1 to 20 features are displayed in Fig. 1a (QDC), b (2NN), and c (Parzen). Overall classification accuracies of all three classifiers with PCA, NWFE, and NWSPMFE are 0.942, 0.949, and 0.961, respectively. NWSPMFE is consistently better than PCA and NWFE in 2NN and Parzen cases across almost all numbers of features. For the QDC classifier, the accuracy of PCA or NWFE is slightly higher than that of NWSPMFE in certain cases. When the number of features equals 1 and 4, the overall classification accuracies of NWFE and NWSPMFE are 0.864 and 0.860 , respectively. When the number of features is 4 , the overall accuracies of PCA and NWSPMFE are 0.959 and 0.956 , respectively. In addition, when the number of features equals $13,15,16$, and 20 , the overall accuracies of

Table 1 Results of kappa analysis for comparison between confusion matrices (DC Mall

\begin{tabular}{|c|c|c|c|c|c|c|c|c|c|c|}
\hline \multirow[t]{2}{*}{ Z Statistic } & & \multicolumn{3}{|l|}{ PCA } & \multicolumn{3}{|c|}{ NWFE } & \multicolumn{3}{|c|}{ NWSPMFE } \\
\hline & & QDC & $2 \mathrm{NN}$ & Parzen & QDC & $2 \mathrm{NN}$ & Parzen & QDC & $2 \mathrm{NN}$ & Parzen \\
\hline \multirow[t]{3}{*}{ PCA } & QDC & 0.000 & & & & & & & & \\
\hline & $2 \mathrm{NN}$ & 0.545 & 0.000 & & & & & & & \\
\hline & Parzen & 0.545 & 0.000 & 0.000 & & & & & & \\
\hline \multirow[t]{3}{*}{ NWFE } & QDC & 0.390 & 0.178 & 0.178 & 0.000 & & & & & \\
\hline & $2 \mathrm{NN}$ & 0.708 & 0.191 & 0.191 & 0.369 & 0.000 & & & & \\
\hline & Parzen & 0.708 & 0.191 & 0.191 & 0.369 & 0.000 & 0.000 & & & \\
\hline \multirow[t]{3}{*}{ NWSPMFE } & QDC & 0.708 & 0.191 & 0.191 & 0.369 & 0.000 & 0.000 & 0.000 & & \\
\hline & $2 \mathrm{NN}$ & 0.875 & 0.388 & 0.388 & 0.566 & 0.198 & 0.198 & 0.198 & 0.000 & \\
\hline & Parzen & 1.047 & 0.593 & 0.593 & 0.770 & 0.403 & 0.403 & 0.402 & 0.205 & 0.000 \\
\hline
\end{tabular}

At the $95 \%$ confidence level; two-sided $\mathrm{Z}$ test, $\mathrm{Z}_{0.95}=1.95996$; $Q D C$ : quadratic Bayes normal classifier; 2NN: 2-nearest neighbor classifier; Parzen: Parzen classifier; PCA: principal component analysis; NWFE: nonparametric weighted feature extraction; NWSPMFE: nonparametric weighted spectral pan-similarity measure feature extraction 


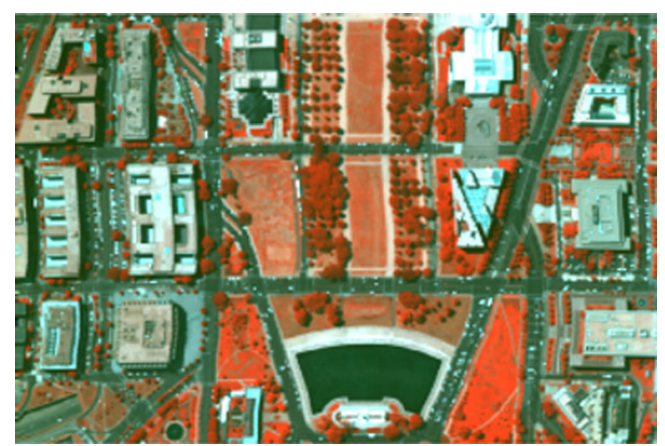

Fig. 3 Three-band false color composite image (D.C. Mall)

PCA and NWFE are 0.945 and 0.938 , respectively. However, the results show that the $2 \mathrm{NN}$ and Parzen classifiers perform better than the QDC classifier for PCA, NWFE, and NWSPMFE. The overall classification accuracies of PCA, NWFE, and NWSPMFE for each classifier are as follows: $0.942,0.947$, and 0.957 , respectively, for $2 \mathrm{NN} ; 0.949,0.957$, and 0.962, respectively, for Parzen; and 0.934, 0.942, and
0.963, respectively, for PCA. The NWSPMFE could reach the maximum classification accuracy $(0.988)$ by using the QDC classifier with 16 extracted features.

The proper number of extracted features is determined by the variations of mean and variance of the minimum distance between transformed divergence and Jeffries-Matusita. Figure 2 displays the mean and variance of the minimum distance between transformed divergence and Jeffries-Matusita for each number of features. Figure 2 clearly shows that the proper number of extracted features is 7 . The drawback of the overall accuracy is that it does not indicate if different classifiers using different features are significantly different. Therefore, Kappa analysis was applied to the first seven features to further evaluate the performance of NWSPMFE. Table 1 presents the results of the Kappa analysis that compares the confusion matrices two at a time to verify if they are significantly different. Despite the higher overall accuracy of NWSPMFE than that of PCA and NWFE, the results of the Kappa analysis show that they are not significantly different

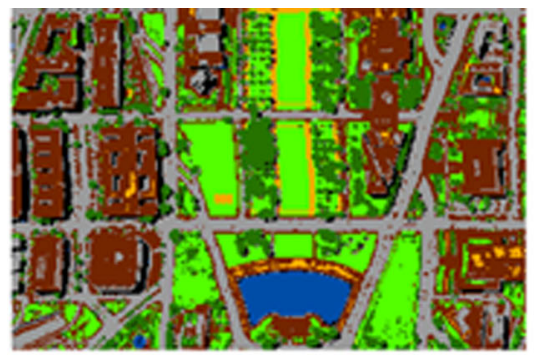

(a).PCA_2NN

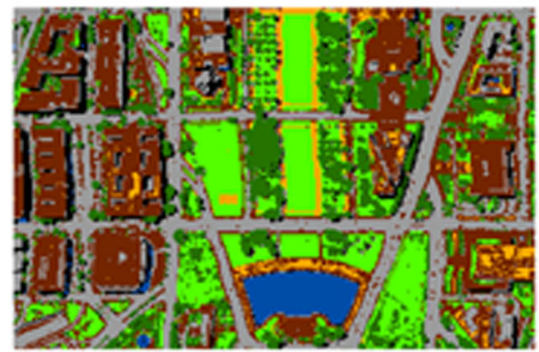

(b).PCA_Parzen

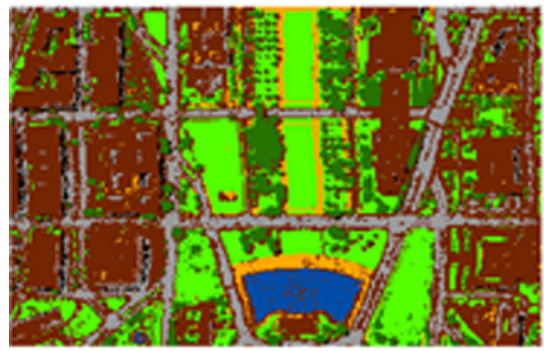

(c).PCA_QDC

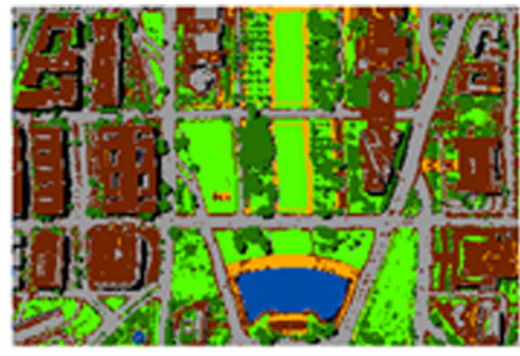

(d).NWFE_2NN

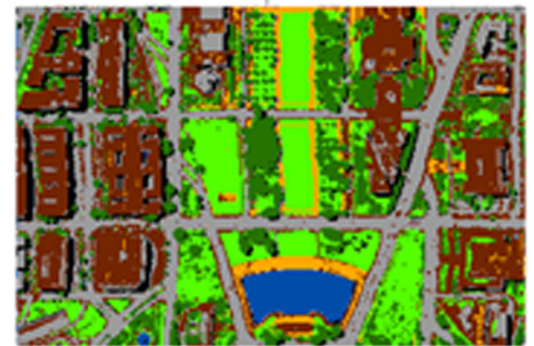

(e). NWFE_Parzen

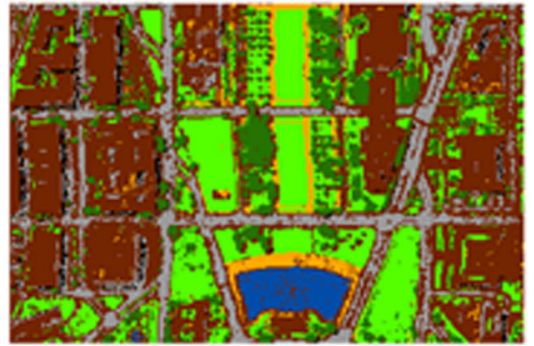

(f). NWFE_QDC

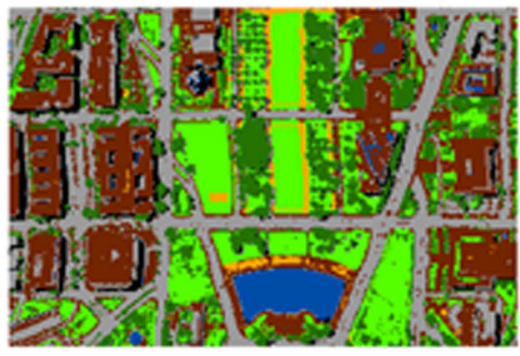

(g). NWSPMFE_2NN

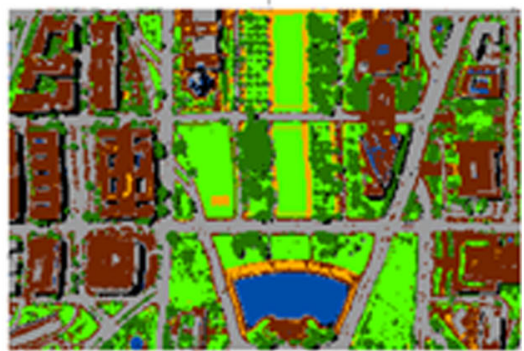

(h). NWSPMFE_Parzen

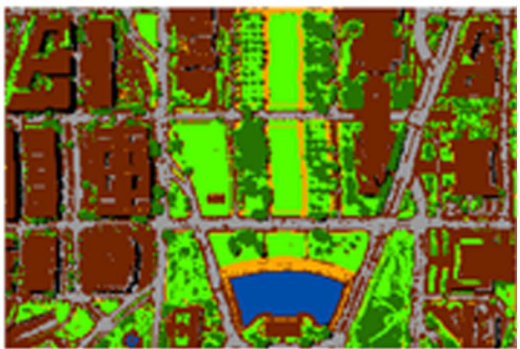

(i). NWSPMFE_QDC

\section{$\square$ roofs $\square$ street $\square$ shadow $\square$ grass $\square$ trees $\square$ patch $\square$ water}

Fig. 4 Classification results of the area of Fig. 2. (a) $P C A+2 \mathrm{NN}$, (b) $P C A+$ Parzen, (c) $P C A+Q D C$, (d) $N W F E+2 \mathrm{NN}$, (e) $N W F E+$ Parzen, (f) $N W F E+Q D C$, (g) $N W S P M F E+2 \mathrm{NN}$, (h) NWSPMFE+Parzen, (i)
NWSPMFE $+Q D C(\mathrm{~L}=7, \mathrm{Ni}=40, \mathrm{P}=7)$, where $\mathrm{L}$ denotes the number of classes, $\mathrm{Ni}$ the number of training samples of each class, and $\mathrm{P}$ the number of bands 
(a)

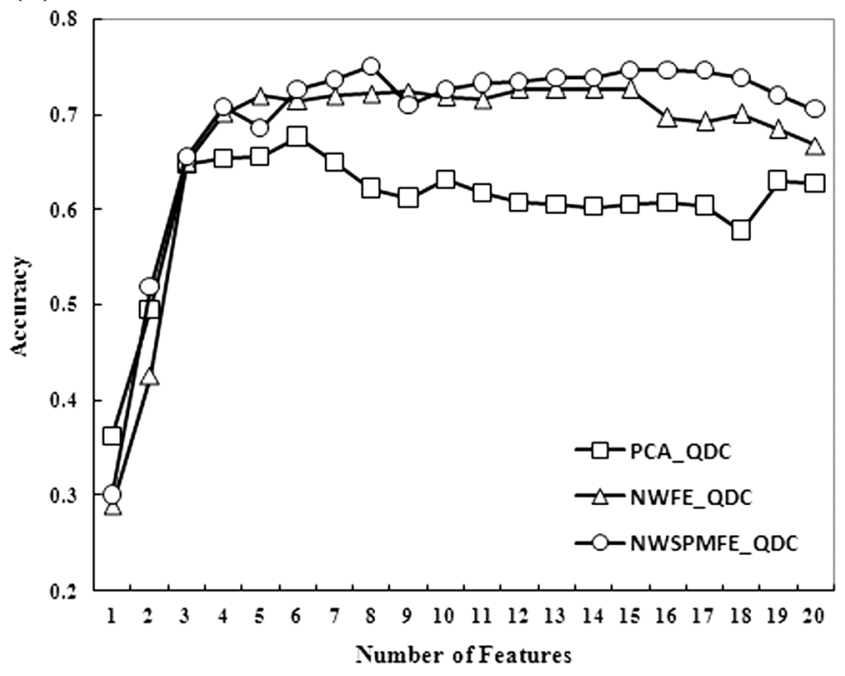

(b)

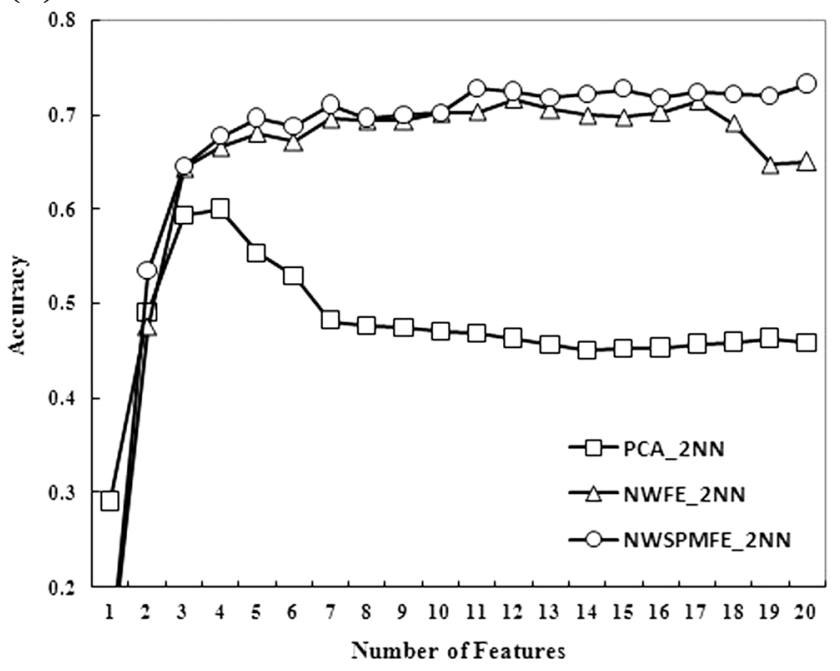

(c)

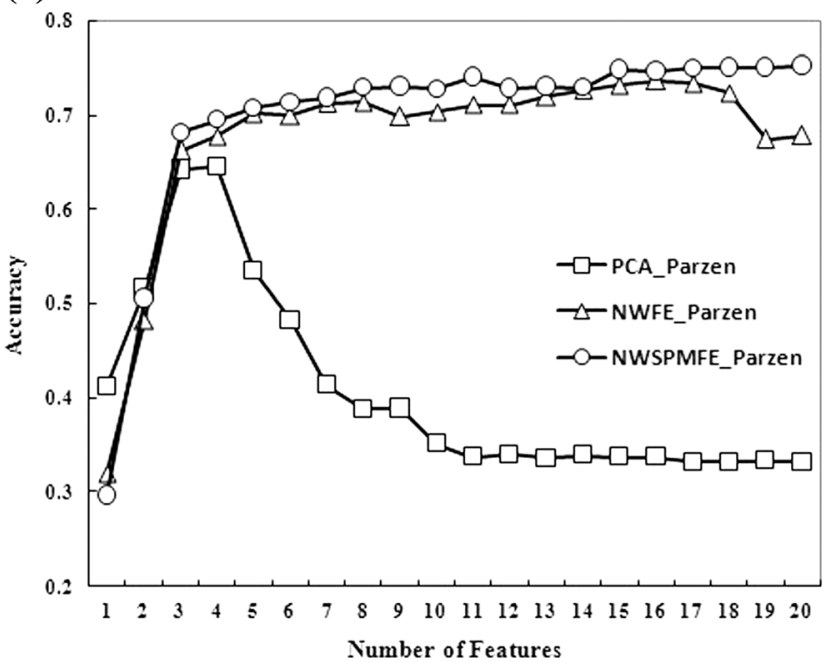

Fig. 5 Overall accuracy versus number of selected features for 3 classifiers (Indian Pine). (a) QDC, (b) 2NN, (c) Parzen
$\left(\mathrm{Z}<\mathrm{Z}_{0.95}\right)$. Fig. 1 and Table 1 clearly indicate that NWFE and NWSPMFE outperformed PCA in all cases. The performance of NWFE is better than that of NWSPMFE in some QDC cases, whereas NWSPMFE outperforms NWFE in $2 \mathrm{NN}$ and Parzen cases.

To better judge the performance of NWSPMFE, visual analysis was applied in this paper. A three-band false-color composite image is shown in Fig. 3 for reference. The D.C. Mall maps that were classified by using $2 \mathrm{NN}$, Parzen, and QDC are displayed in Fig. 4a, b and c (PCA), d, e and $\mathrm{f}$ (NWFE), and g, h and i (NWSPMFE), respectively. Figure 4 shows the following: (1) All three classifiers with NWSPMFE features performed better than the classifiers with PCA or NWFE features. (2) NWSPMFE performed better than PCA or NWFE in almost in all classes, especially roofs, patch, water, and shadow classes. (3) For these three classifiers, NWFE performs better than PCA. Two phenomena were observed in the classification results of PCA, NWFE, and NWSPMFE: same spectrum with different objects and same object with different spectrums. The classes that exist in the same spectrum with different objects are mainly roofs and street and roofs and patch. The class that exists in the same object with different spectrum is mainly grass. Roofs and street and roofs and patch have been misclassified in the classification results, but NWSPMFE results are superior to other results. The smoothness of classification results obtained by using NWSPMFE features is also better than that of other classified results.

\section{Results of Indian Pine Dataset}

The overall accuracies using 1-20 PCA, NWFE, and NWSPMFE features of the Indian Pine AVIRIS imagery are displayed in Fig. 5a (QDC), b $(2 \mathrm{NN})$, and $\mathrm{c}$ (Parzen). The overall classification accuracies of all three classifiers with NWSPMFE, NWFE and PCA are $0.697,0.665$, and 0.496 , respectively. The average overall accuracies for QDC, $2 \mathrm{NN}$, and Parzen classifiers using the different features are as follows: 0.605, 0.477 , and 0.407 , respectively, using 1 to 20 PCA features; $0.671,0.649$, and 0.677 , respectively, using NWFE; and 0.700, 0.686, and 0.704, respectively, using NWSPMFE. The best classification accuracy is 0.776 and is obtained by the Parzen classifier with 15 features extracted by NWSPMFE.

Figure 6 presents a comparison of the mean and variance of the minimum distance between transformed divergence and Jeffries-Matusita for the Indian Pine dataset. Only 14 features are available due to the nature of the two distances and the least sample size. Obviously, choosing 13 features as an example to further discuss the performance of NWSPMFE is appropriate. Table 2 presents the result of the Kappa analysis that compares the confusion matrices two at a time to 
Fig. 6 Mean and variance of the minimum value between transformed divergence and Jeffries-Matusita distances using 2-14 features (Indian Pine)

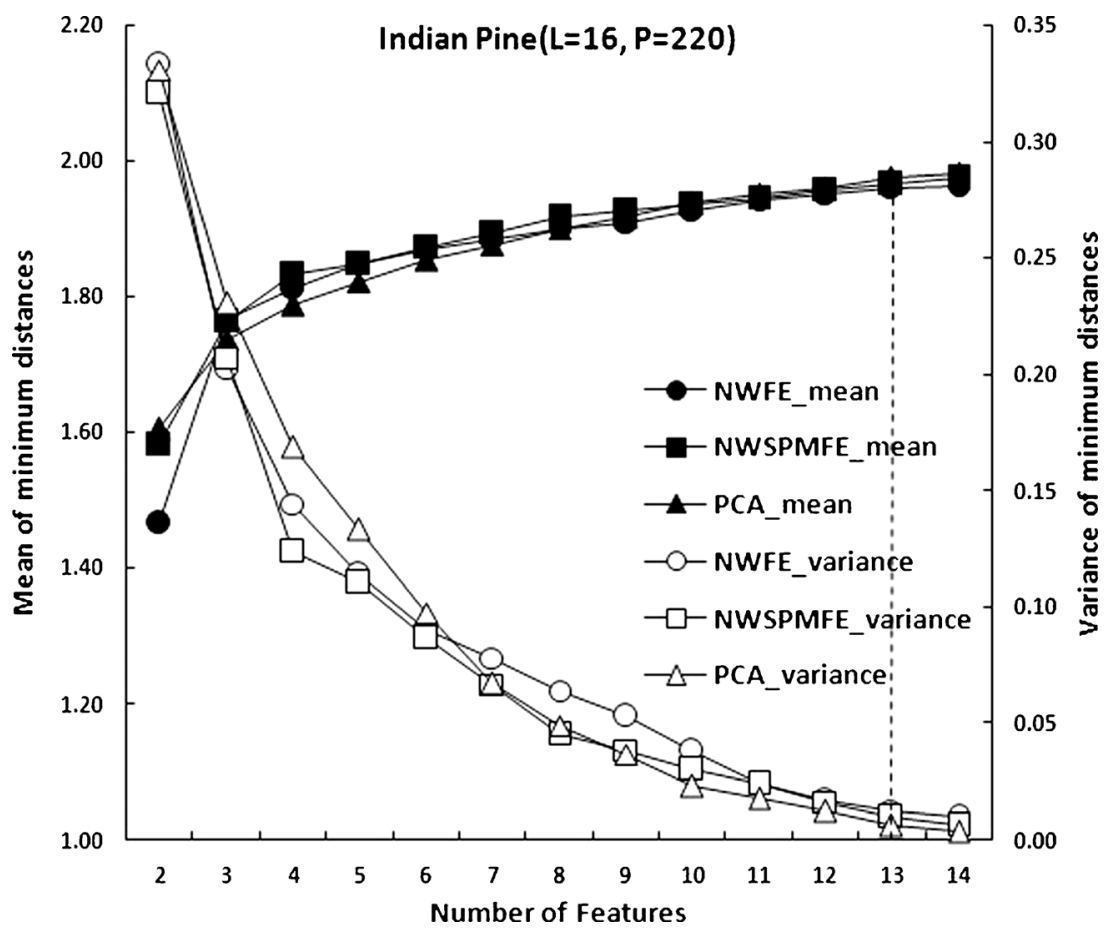

determine if they are significantly different. The result shows a significant difference between PCA and other algorithms ( $\mathrm{Z}>$ $Z_{0.95}$ ). Despite the $3.2 \%$ higher overall accuracy of NWSPMFE than NWFE, the results of the Kappa analysis show that NWFE and NWSPMFE are not significantly different $\left(\mathrm{Z}<\mathrm{Z}_{0.95}\right)$. Fig. 5 and Table 2 clearly show that NWSPMFE and NWFE performed better than PCA in all cases. Although NWFE and NWSPMFE are not significantly different, all three classifiers with NWSPMFE features performed better than the classifiers with NWFE features in almost all cases.

\section{Conclusions}

In this paper, we proposed NWSPMFE, which quantifies three spectral features by replacing ED in NWFE with SPM.

The main contribution of the paper is as follows:

1 SPM is better for quantifying differences between two spectral vectors than ED. The main reason for its superiority is that SPM takes into account three spectral features (spectral vector magnitude, spectral vector direction, and spectral information content), while ED quantifies the

Table 2 Results of Kappa analysis for comparison between confusion matrices for Indian Pine dataset

\begin{tabular}{|c|c|c|c|c|c|c|c|c|c|c|}
\hline \multirow[t]{2}{*}{ Z Statistic } & & \multicolumn{3}{|l|}{ PCA } & \multicolumn{3}{|c|}{ NWFE } & \multicolumn{3}{|c|}{ NWSPMFE } \\
\hline & & QDC & $2 \mathrm{NN}$ & Parzen & QDC & $2 \mathrm{NN}$ & Parzen & QDC & $2 \mathrm{NN}$ & Parzen \\
\hline \multirow[t]{3}{*}{ PCA } & QDC & 0.000 & & & & & & & & \\
\hline & $2 \mathrm{NN}$ & 2.212 & 0.000 & & & & & & & \\
\hline & Parzen & 4.887 & 2.825 & 0.000 & & & & & & \\
\hline \multirow[t]{3}{*}{ NWFE } & QDC & 2.731 & 4.675 & 6.869 & 0.000 & & & & & \\
\hline & $2 \mathrm{NN}$ & 2.680 & 4.639 & 6.854 & 0.063 & 0.000 & & & & \\
\hline & Parzen & 3.146 & 4.993 & 7.052 & 0.492 & 0.554 & 0.000 & & & \\
\hline \multirow[t]{3}{*}{ NWSPMFE } & QDC & 3.179 & 5.015 & 7.061 & 0.534 & 0.597 & 0.043 & 0.000 & & \\
\hline & $2 \mathrm{NN}$ & 3.133 & 4.984 & 7.050 & 0.474 & 0.537 & 0.018 & 0.061 & 0.000 & \\
\hline & Parzen & 3.223 & 5.047 & 7.078 & 0.587 & 0.649 & 0.096 & 0.053 & 0.114 & 0.000 \\
\hline
\end{tabular}

At the $95 \%$ confidence level; two-sided $\mathrm{Z}$ test, $\mathrm{Z}_{0.95}=1.95996 ;$ $Q D C$ : quadratic Bayes normal classifier; 2NN: 2-nearest neighbor classifier; Parzen: Parzen classifier; PCA: principal component analysis; NWFE: nonparametric weighted feature extraction; NWSPMFE: nonparametric weighted spectral pan-similarity measure feature extraction 
magnitude differences only. This finding answers the question of why SPM is superior to ED.

2 For the NWFE algorithm, determining or defining the weight function is the most important step. The weight function based on the ED in NWFE is used to assign different weights to every sample to compute the weighted mean. This paper replaced ED with SPM because ED captures the differences between two spectra better than SPM.

3 The performances of all three classifiers that use NWSPMFE and NWFE features are better than those of classifiers that use PCA features. Particularly for the Indian Pine dataset, NWSPMFE and NWFE performed significantly better than PCA based on the results of the Kappa analysis and overall accuracy measure.

4 For the two hyperspectral datasets (D.C. Mall and Indian Pine), despite the results of the Kappa analysis, NWSPMFE and NWFE are not significantly different, and NWSPMFE's runtime is somewhat longer than that of NWFE (110.56 and $102.43 \mathrm{~s}$ for the D.C. Mall dataset, and 60.16 and $55.60 \mathrm{~s}$ for the Indian Pine dataset) due to the computational complexity of NWSPMFE. However, the overall accuracy of NWSPMFE is superior to that of NWFE in almost all cases. Therefore, given the choice of only these two algorithms, NWSPMFE should be selected because its slightly longer runtime that that of NWFE is not the deciding factor.

Acknowledgments This work was supported by the National Natural Science Foundation of China under grant number 2012BAC21B01 and the Geological Survey program of China Geological Survey under grant number GZH201200503. We thank Prof. Landgrebe and Biehl for providing the AVIRIS and HYDICE data. We would also like to thank 37 Steps website for providing the Pattern Recognition Tools (PRTools). Many thanks go to the anonymous reviewers for the comments on the manuscript. We would also like to thank "Data Sharing Network of Earth System Science".

\section{References}

Carvalho Júnior, O. A., Guimarães, R. F., Gillespie, A. R., Silva, N. C., \& Gomes, R. A. (2011). A New Approach to Change Vector Analysis Using Distance and Similarity Measures. Remote Sensing, 3, $2473-$ 2493.

Chang, Chein-I., 2000.“An information-theoretic approach to spectral variability, similarity, and discrimination for hyperspectral image analysis." Review of. Information Theory, IEEE Transactions on 46 (5):1927-32.

Chen, Bin, Anthony Vodacek, and Nathan D Cahill., 2012. Novel spectral similarity measure for high resolution urban scenes. Paper presented at the Geoscience and Remote Sensing Symposium (IGARSS), 2012 I.E. International.

Chein-Chi, C., Du, Y., Chein-I, C., Hsuan, R., Jensen, J. O., \& D’Amico, F. M. (2004). New hyperspectral discrimination measure for spectral characterization. Review of. Optical Engineering, 43(8), 17771786 .
Congalton, R. G. (1991). A review of assessing the accuracy of classifications of remotely sensed data. Remote sensing of environment, 37(1), 35-46.

Das, P., Moll, M., Stamati, H., Kavraki, L. E., \& Clementi, C. (2006). Low-dimensional, free-energy landscapes of protein-folding reactions by nonlinear dimensionality reduction. Review of. Proceedings of the National Academy of Sciences, 103(26), 9885-9890.

De Maesschalck, R., Jouan-Rimbaud, D., \& Massart, D. L. (2000). The mahalanobis distance. Chemometrics and intelligent laboratory systems, 50(1), 1-18.

Duda, R. O., Hart, P. E., \& Stork, D. G. (2012). Pattern classification. New York: Wiley.

Duin, R. (2003). Prtools version 3.2: A matlab toolbox for pattern recognition. Delft: Pattern Recognition Group, Delft University of Technology.

Duin, R. P., \& E. Pekalska. (2007). The science of pattern recognition. Achievements and perspectives. Challenges for Computational Intelligence, Springer: 221-259.

Foody, G. M. (1992). On the compensation for chance agreement in image classification accuracy assessment. Photogrammetric Engineering and Remote Sensing, 58(10), 1459-1460.

Foody, G. M. (2002). Status of land cover classification accuracy assessment. Remote sensing of environment, 80(1), 185-201.

Friedman, J. H. (1989). Regularized discriminant analysis. Review of. Journal of the American statistical association, 84(405), 165-175.

Howarth, P. J., Marceau, D. J., \& Gong, P. (1992). A comparison of spatial feature extraction algorithms for land-use classification with SPOT HRV data. Review of. Remote Sensing of Environment, 40(2), $137-151$.

Hastie, T., Tibshirani, R., \& Friedman, J. (2009). The Elements of Statistical Learnin. New York: Springer.

Hsieh, Pi-Fuei, \& David Landgrebe (1998). "Classification of high dimensional data." Review of. ECE Technical Reports:52.

Hughes, G. (1968). "On the mean accuracy of statistical pattern recognizers." Review of. Information Theory, IEEE Transactions on, 14(1), 55-63.

Kong, X., Ning, S., Jianbin, T., \& Yan, G. (2012). A new spectral similarity measure based on multiple features integration. In Review of. Spectroscopy and Spectral Analysis (31st ed., Vol. 8, pp. 70-2166).

Kuo, Bor-Chen, Li-Wei Ko, Chia-Hao Pai, \& DA Landgrebe (2003). Regularized feature extractions for hyperspectral data classification. Paper presented at the Geoscience and Remote Sensing Symposium, 2003. IGARSS'03. Proceedings.2003 I.E. International.

Kuo, Bor-Chen, \& David A Landgrebe (2002a). Hyperspectral data classification using nonparametric weighted feature extraction. Paper presented at the Geoscience and Remote Sensing Symposium, 2002. IGARSS`02.2002 I.E. International.

Kuo, B.-C., \& Landgrebe, D. A. (2004). Nonparametric weighted feature extraction for classification. Review of. Geoscience and Remote Sensing, IEEE Transactions on, 42(5), 1096-1105.

Kuo, B.-C., \& C.-H. Li (2005). Kernel nonparametric weighted feature extraction for classification. AI 2005: Advances in Artificial Intelligence, Springer: 567-576.

Landgrebe, D. A., \& Kuo, B.-C. (2002). A robust classification procedure based on mixture classifiers and nonparametric weighted feature extraction. Geoscience and Remote Sensing, IEEE Transactions on, 40(11), 2486-2494.

Landgrebe, D. A. (2003). "Signal Theory Methods in Multispectral Remote Sensing. "In. New York: Wiley.

Liu, C., Frazier, P., \& Kumar, L. (2007). Comparative assessment of the measures of thematic classification accuracy. Remote sensing of environment, 107(4), 606-616.

Ma, Z., \& Redmond, R. L. (1995). Tau coefficients for accuracy assessment of classification of remote sensing data. Photogrammetric Engineering and Remote Sensing, 61(4), 435-439. 
Naresh Kumar, M., Seshasai, M. V. R., Vara Prasad, K. S., Kamala, V., Ramana, K. V., Dwivedi, R. S., \& Roy, P. S. (2011). A new hybrid spectral similarity measure for discrimination among Vigna species. Review of. International journal of Remote sensing, 32(14), 40414053.

Paclik, P., \& Duin, R. P. (2003). Dissimilarity-based classification of spectra: computational issues. Real-Time Imaging, 9(4), 237-244.

Parzen, E. (1962). On estimation of a probability density function and mode. The annals of mathematical statistics, 33(3), 1065-1076.

Pękalska, E., \& Duin, R. P. (2002). Dissimilarity representations allow for building good classifiers. Pattern Recognition Letters, 23(8), 943-956.

Pekalska, E., Paclik, P., \& Duin, R. P. (2001). A generalized kernel approach to dissimilarity-based classification. The Journal of Machine Learning Research, 2, 175-211.

Raudys, Š. \& A. Saudargiene (1998). Structures of the covariance matrices in the classifier design. Advances in Pattern Recognition, Springer: 583-592.

Rayens, W., \& Greene, T. (1991). Covariance pooling and stabilization for classification. Computational Statistics \& Data Analysis, 11(1), 17-42.

Schowengerdt, R. A. (2006). Remote sensing: models and methods for image processing. Waltham: Academic.

Smits, P., Dellepiane, S., \& Schowengerdt, R. (1999). Quality assessment of image classification algorithms for land-cover mapping: a review and a proposal for a cost-based approach. International journal of remote sensing, 20(8), 1461-1486.

Stehman, S. V. (1997). Selecting and interpreting measures of thematic classification accuracy. Remote sensing of Environment, 62(1), 77-89.
Stehman, S. V., \& Czaplewski, R. L. (1998). Design and analysis for thematic map accuracy assessment: fundamental principles. Remote Sensing of Environment, 64(3), 331-344.

Story, M., \& Congalton, R. G. (1986). Accuracy assessment-A user's perspective. Photogrammetric Engineering and remote sensing, 52(3), 397-399.

Sun, Z.-L., Huang, D.-S., \& Cheun, Y.-M. (2005). Extracting nonlinear features for multispectral images by FCMC and KPCA. Review of. Digital Signal Processing, 15(4), 331-346.

Sweet, James Norman (2003). The spectral similarity scale and its application to the classification of hyperspectral remote sensing data. Paper presented at the Advances in Techniques for Analysis of Remotely Sensed Data, 2003 I.E. Workshop on.

Tang, Y. Y., Tao, Y., \& Lam, E. (2002). New method for feature extraction based on fractal behavior. Review of. Pattern recognition, 35(5), 1071-1081.

Tian, YanQin, Ping Guo, \& Michael R Lyu (2005). Comparative studies on feature extraction methods for multispectral remote sensing image classification. Paper presented at the Systems, Man and Cybernetics, 2005 I.E. International Conference on.

van der Meer, F., \& Bakker, W. (1997). Cross correlogram spectral matching: Application to surface mineralogical mapping by using AVIRIS data from Cuprite, Nevada. Review of. Remote Sensing of Environment, 61(3), 371-382.

Webb, A. R. (2002). Statistical pattern recognition. New York: Wiley.

Young, Tzay Y., \& King Sun Fu (1986). Handbook of pattern recognition and image processing: Academic press Orlando. 